\title{
PA-007 VARIABILITY IN CLINICAL RESEARCH DATA MANAGEMENT PRACTICES: LESSONS FROM THE MALARIA COMMUNITY
}

Amélie Julé, ${ }^{1}$ Hazel Ashurst, ${ }^{2}$ Laura Merson, ${ }^{2}$ Piero Olliaro, ${ }^{3}$ Vicki Marsh, ${ }^{1}$ Trudie Lang, ${ }^{4}$ Philippe Guérin ${ }^{2}$. ${ }^{1}$ Nuffield - University of Oxford, United Kingdom; ${ }^{2}$ WWARN, United Kingdom; ${ }^{3}$ UNICEF-UNDP-World Bank-WHO-TDR, Switzerland;

${ }^{4}$ TGHN, University of Oxford, United Kingdom

\subsection{6/bmjgh-2016-000260.46}

Background Appropriate data management (DM) is critical to produce valuable research data, especially with growing prospects for long-term archiving, sharing and individual patient data meta-analysis. The experience of the WorldWide Antimalarial Resistance Network (WWARN) in handling data from clinical studies is that DM practices vary greatly, which affects curation and optimal use of shared data. Our work explores how clinical trial data are usually managed and why this varies. We aim to understand the needs in DM systems (DMS) for resource-limited research settings within low- and middle-income countries (LMICs).

Methods Using published literature and discussions with key informants, we developed a semi-quantitative instrument to assess the robustness of the initial DMS and the resulting 're-usability' of clinical research data. We also defined study covariates which could account for the observed variability (e.g. type of sponsor/funding, partners involved, trial phase). The strength of correlations between indicators of good DM practices, resulting data quality and study context will be tested through statistical modelling.

Results The instrument covers the following dimensions of data robustness: meta-data availability, comprehensiveness and exhaustiveness; dataset completeness; and data accuracy. It is currently being piloted on a subset of 20 studies (about $5 \%$ of the total WWARN database), to test its applicability in highlighting DM practices' variations and in capturing other relevant study characteristics. After finalisation of the instrument, the analysis will be rolled out to 150 studies. We will present the patterns and correlations between specific indicators and study covariates we observe within this randomly selected sample, and discuss their implications in terms of DM capacity-strengthening.

Conclusions The significance of quantitative findings will be challenged using qualitative interviews and visits at institutions for in-depth case studies of DM practices. Results of the overall mixed-methods work could inform strategies for clinical research DM capacity-strengthening in LMICs, including initiatives relevant to the European \& Developing Countries Clinical Trials Partnership (EDCTP). 